\begin{tabular}{|c|c|}
\hline$-1--$ & Appareil \\
\hline pru & $\begin{array}{l}16 \mid 2015 \\
\text { Individuer Simondon. De la redécouverte aux } \\
\text { prolongements }\end{array}$ \\
\hline
\end{tabular}

\title{
Techno-esthétiques ou philosophies de l'interaction : les réflexions de Gilbert Simondon et John Dewey
}

\section{Elisa Binda}

\section{OpenEdition \\ Journals}

\section{Édition électronique}

URL : https://journals.openedition.org/appareil/2217

DOI : 10.4000/appareil.2217

ISSN : 2101-0714

\section{Éditeur}

MSH Paris Nord

\section{Référence électronique}

Elisa Binda, «Techno-esthétiques ou philosophies de l'interaction : les réflexions de Gilbert Simondon et John Dewey », Appareil [En ligne], 16 | 2015, mis en ligne le 09 février 2016, consulté le 21 septembre 2021. URL : http://journals.openedition.org/appareil/2217 ; DOI : https://doi.org/10.4000/ appareil.2217

Ce document a été généré automatiquement le 21 septembre 2021.

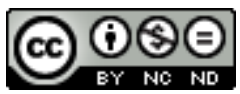

Appareil est mis à disposition selon les termes de la Licence Creative Commons Attribution - Pas d'Utilisation Commerciale - Pas de Modification 4.0 International. 


\title{
Techno-esthétiques ou philosophies de l'interaction : les réflexions de Gilbert Simondon et John Dewey
}

\author{
Elisa Binda
}

\section{Introduction}

1 Le but de cet article est de montrer une possible analogie entre la techno-esthétique de Gilbert Simondon et ce que l'on peut appeler une réflexion de type techno-esthétique au sein de la pensée de John Dewey. La présence d'une telle analogie peut sembler relever du hasard, puisque Simondon et Dewey appartiennent à des traditions philosophiques différentes. Mais ce qui importe ici est de montrer une possible convergence théorique entre eux, à partir de leur capacité commune à esquisser une forme non conventionnelle d'esthétique. Cela est de la plus grande importance, à l'époque où la technique est devenue omniprésente et détermine notre sensibilité ellemême.

2 La lettre que Gilbert Simondon adresse à Jacques Derrida en 1982 peut être regardée comme l'esquisse d'une reconfiguration de l'esthétique en direction de ce que Simondon définit comme une techno-esthétique. Il faut considérer la lettre Sur la technoesthétique comme une contribution fondamentale à la réflexion contemporaine sur l'esthétique. L'hypothèse que je souhaite formuler relève d'une considération de l'esthétique comme philosophie "non spéciale ", qui ne se limite pas à analyser le type d'expérience lié aux beaux-arts ${ }^{1}$, mais devient une réflexion sur toute l'expérience en tant que telle, en thématisant le mode spécifique sur lequel le genre humain, via sa sensibilité, interagit avec le monde ${ }^{2}$. Nous remontons ici au sens originaire du mot " esthétique », au grec aesthesis qui signifie sensibilité, sensation. À ce propos, il est significatif que Simondon lui-même ait employé ce terme grec dans sa lettre : cet usage n'apparaît pas au sein de la réflexion que le philosophe avait déjà consacrée à la question esthétique dans $\mathrm{Du}$ mode d'existence des objets techniques (1958). Comme le 
suggère le titre, l'enquête de Simondon "sur la techno-esthétique " concerne la relation étroite entre le domaine technique et le domaine esthétique. Cette relation, dont Simondon s'occupe pourtant déjà dans Du mode d'existence des objets techniques, s'avère encore plus stricte dans la lettre à Derrida, qui s'achève sur une affirmation décisive :

[...] le sentiment techno-esthétique semble être une catégorie plus primitive que le sentiment esthétique seul ou l'aspect technique considéré sous l'angle de la seule fonctionnalité, qui est appauvrissante ${ }^{3}$.

3 Considérer la catégorie du sentiment techno-esthétique comme plus primitive que celle du sentiment esthétique seul revient à reconnaitre que l'aesthesis, dans son fond, a toujours été connectée avec les éléments techniques qui nous permettent d'articuler notre relation sensible au monde. Nous assistons ici à l'émergence, dans la philosophie simondonienne, d'une esthétique qui pose au centre de sa réflexion l'interaction entre la sensibilité du corps humain et ce que cette sensibilité rencontre, reçoit et élabore.

4 Le parallèle que je souhaite établir entre Simondon et Dewey concerne le type d'expérience esthétique exposé dans le texte Sur la techno-esthétique, dans la mesure où cette expérience esthétique n'est pas sans lien avec celle pensée par John Dewey (1859-1952) dans certains de ses écrits principaux : Experience and Nature (1925); Art as Experience (1934); Knowing and the Known (1949).

5 Les deux philosophes ont élaboré leurs réflexions à partir de la reconnaissance d'un fort noyau interactionnel agissant à des niveaux différents de l'expérience : au niveau des interactions se produisant entre un organisme et son milieu, aussi bien qu'au niveau des rapports que l'homme entretien avec ses propres artefacts. La richesse de la réflexion techno-esthétique est liée à de telles questions. Avant de définir, au travers leurs similitudes, les esthéto-techniques qui caractérisent les philosophies de Simondon et de Dewey, je voudrais faire quelques remarques pour montrer comment, dès la description de la relation de base entre organisme et milieu, on peut déjà relever, chez les deux auteurs, des points conceptuels communs.

\section{Organisme et milieu, l'origine de l'interaction : « transduction » et « transaction »}

6 Quel est le type de relation que l'organisme entretient avec son milieu? Tandis qu'entre Simondon et Dewey il existe des différences remarquables relatives aux différents contextes et cadres de référence philosophiques qui sont les leurs ${ }^{4}$, les deux philosophes offrent néanmoins des réponses qui se rapprochent de manière évidente. Tout d'abord, leur intention commune est de montrer comment cette relation entre organisme et milieu n'est ni une simple inclusion ni une forme d'adaptation passive, mais qu'elle est, tout au contraire, à l'origine de processus transformateurs aussi bien pour l'individu que pour le milieu. Simondon et Dewey reconnaissent entre organisme et milieu un rapport que l'on peut qualifier de co-constitution. Il en résulte que c'est la relation elle-même qui joue un rôle constitutif par rapport à ce qu'elle met en connexion. Dans L'individuation à la lumière des notions de forme et d'information, Simondon montre que «ce que l'individuation fait apparaitre n'est pas seulement l'individu mais le couple individu-milieu ${ }^{5} »$. Ce que Jean-Hugues Barthélémy et Vincent Bontems commentent en ces termes : 
[...] l'individuation, qu'elle soit genèse radicale de l'individu physique ou vivant ou qu'elle soit l'« individualisation » de l'individu vivant déjà individué, consiste en un déphasage des potentiels en individu et son milieu associé avec lequel il entretient des relations énergétiques qui déterminent l'actualisation (partielle ou complète) de ses potentiels ${ }^{6}$.

7 Pour comprendre comment il existe, entre individu et milieu, une communication et une modulation continues, il faut penser à la manière dont Simondon utilise le concept de transduction mais aussi celui, prélevé au domaine de la physique, de champ. Dans L'individuation à la lumière des notions de forme et d'information, Simondon soutient que l'opération transductive est une individuation in progress :

[elle] correspond à cette existence de rapports prenant naissance lorsque l'être préindividuel s'individue ; elle exprime l'individuation et permet de la penser ; [...] elle s'applique à l'ontogénèse et est l'ontogénèse même ${ }^{7}$.

Il est donc évident que, dès lors que l'individu s'individue à partir du préindividuel, une série de "rapports prégnants ", qui mettent l'individu en communication active avec son milieu, s'engendre immédiatement. En s'autorisant d'un dialogue constant avec les disciplines scientifiques de son époque, Simondon formule à cet égard une hypothèse « analogue à celle des quanta en physique, analogue aussi à celle de la relativité des niveaux d'énergie potentielle [...]. Selon cette hypothèse, il serait possible de considérer toute véritable relation comme ayant rang d'être, et comme se développant à l'intérieur d'une individuation nouvelle; la relation ne jaillit pas entre deux termes qui seraient déjà individus; elle est un aspect de la résonance interne d'un système d'individuation; elle fait partie d'un état de système $e^{8}$ ". Par conséquent, la relation joue un rôle constitutif par rapport à ce qu'elle relie. Elle n'est jamais externe mais interne, constitutive de l'individualité même : ce que Simondon définit comme transduction est une opération relationnelle dont les termes ne préexistent pas à son effectuation : ils émergent dans le processus même. En empruntant les mots de Didier Debaise, nous pouvons dire que les relations ne sont pas des "modalités d'existence", elles sont constitutives de l'existence même: c'est donc une véritable ontologie de la relation. Le modèle qui permet à Simondon de concevoir l'être comme relation est donné par la notion de champ :

[...] elle est un présent fait aux sciences humaines par les sciences de la nature. Elle établit une réciprocité de statuts ontologiques et de modalités opératoires entre le tout et l'élément. En effet, dans un champ, quel qu'il soit, électrique, électromagnétique, de gravité, ou de n'importe quelle autre espèce, l'élément possède deux statuts et remplit deux fonctions : $1^{\circ}$ en tant que recevant l'influence du champ, il est soumis aux forces du champ; il est en un certain point du gradient par lequel on peut représenter la répartition du champ ; $2^{\circ}$ il intervient dans le champ à titre créateur et actif, en modifiant les lignes de force du champ sans définir ce qu'il y a en tel point ${ }^{9}$.

Dans Knowing and the Known, Dewey utilise également des formulations marquées par la science de son époque, en faisant spécialement référence, comme le fait Simondon, à la physique et à la biologie. Mais Dewey utilise aussi le concept de transaction, qui répond à la tentative de trouver un vocable permettant d'attirer l'attention sur le système organisme-milieu pris dans son ensemble, au lieu de privilégier l'un de ses pôles. Avant d'expliquer en détail ce concept de transaction, il en donne une première définition générale :

[...] in our general procedure of inquiry no radical separation is made between that which is observed and the observer in the way which is common in the epistemologies and in standard psychologies and psychological constructions. 
Instead, observer and observed are held in close organisation. [...] Our position is simply that since man as an organism has evolved among other organisms in an evolution called "natural ", we are willing under hypothesis to treat all of his behavings, including his most advanced knowings, as activities not of himself alone, but as processes of the full situation of organism-environment ${ }^{10}$. affirme explicitement que l'organisme et son milieu forment un tout unique, au point que nul comportement de l'organisme ne peut être conçu comme l'œuvre du seul sujet ; il est plutôt un processus qui appartient au système organisme-milieu dans son ensemble. " auto-action », « inter-action » et "trans-action ». Ils constituent, selon Dewey, les modes historiques de représentation de la manière dont l'organisme se rapporte au monde. Voici la description que le philosophe donne de chacun de ces trois niveaux : 2. Il y a Inter-actic
interconnection »; y a Trans-action «where systems of description and naming are employed to dea with aspects and phases of action, without final attribution to "elements" or other presumptively detachable or independent "entities", "essences", or "realities", and without isolation of presumptively detachable "relations" from such detachable "elements" "11. 'exemple le plus évident d'application du point de vue «auto-actionnel » est la physique aristotélicienne, construite autour de la notion de «substance ». L'abandon du modèle de l'auto-action se produit avec le travail de Galilée, qui pose les bases à partir desquelles se développera la perspective inter-actionnelle, achevée par Newton. Il faut attendre Einstein, selon Dewey, pour parvenir finalement à l'application du critère trans-actionnel en physique.

Après avoir montré comment ces trois niveaux - auto-action, inter-action et trans-action - sont appliqués au domaine de la physique, Dewey porte son attention sur la biologie. Il y montre les différences existant entre interaction et transaction. Tandis qu'une perspective interactionnelle considère l'organisme et le milieu comme existant l'un indépendamment de l'autre, l'interaction étant un troisième élément intervenant entre les deux premiers, un point de vue transactionnel suppose que les trois éléments sont considérés et observés en tant que parties d'un seul système. Si, dans l'interaction, on suppose que l'organisme et les objets de son milieu sont présents comme formes d'existence essentiellement séparées, antécédentes à leur mise en relation :

[...] the Transaction assumes no pre-knowledge of either organism or environment alone as adequate, not even as respects the basic nature of current conventional distinctions between them, but requires their primary acceptance in common system, with full freedom reserved for their developing examination ${ }^{12}$.

Dewey souligne donc que l'organisme et le milieu ne peuvent d'aucune manière être considérés comme séparés l'un de l'autre ${ }^{13}$.

Dans les réflexions de Simondon et de Dewey, il apparaît ainsi que le milieu n'est pas quelque chose d'extérieur aux activités de l'organisme ; le milieu est leur élément, il est milieu au sens où il est, dans le même temps, élément intermédiaire dans l'exécution et l'achèvement des activités des organismes, ainsi que le moyen à travers lequel ces opérations se réalisent. Ceci est vrai de manière encore plus significative et complexe 
pour ce qui concerne l'agir de l'être humain. En conclusion de Knowing and the Known, Dewey écrit :

[...] the human life itself, both severally and collectively, consists of transactions in which human beings partake together with non-human things of the milieu along with other human beings, so that without this togetherness of human and nonhuman partakers we could not even stay alive, to say nothing of accomplishing anything. From birth to death every human being is a Party, so that neither he nor anything done or suffered can possibly be understood when it is separated from the fact of participation in an extensive body of transactions - to which a given human being may contribute and which he modifies, but only in virtue of being a partaker in them ${ }^{14}$.

19 Il paraît évident que nous pouvons substituer le terme deweyen de transaction au terme simondonien de transduction sans altérer d'aucune manière le sens du passage précédemment cité.

\section{Techno-esthétiques : confronter Simondon et Dewey}

Le caractère trans-actif du rapport entre organisme et milieu, si présent dans les philosophies de Simondon et Dewey, a des conséquences importantes sur la question de la technique dans son rapport intrinsèque avec l'esthétique. Rappelons le noyau théorique de la réflexion techno-esthétique de Simondon: "le sentiment technoesthétique semble être une catégorie plus primitive que le sentiment esthétique seul ou l'aspect technique considéré sous l'angle de la seule fonctionnalité, qui est appauvrissante ». Dans son texte L'Art comme expérience, Dewey déclare de son côté qu " afin de comprendre l'esthétique dans ses formes accomplies et reconnues, on doit commencer à la chercher dans la matière brute de l'expérience ${ }^{15} »$. Or, il fournit l'exemplification de cette présence diffuse de l'esthétique dans la vie quotidienne à travers une longue suite d'images qui mettent fortement en valeur le rapport de l'homme à la technique :

[...] la voiture de pompiers passant à toute allure, les machines creusant d'énormes trous dans la terre, la silhouette d'un homme, aussi minuscule qu'une mouche, escaladant la flèche du clocher, les hommes perchés dans les airs sur des poutrelles, lançant et rattrapant des tiges de métal incandescent. [...], la grâce alerte du joueur de ballon ${ }^{16}$.

Ces exemples trouvent leurs analogues dans "Sur la techno-esthétique ", où Simondon dresse une liste de situations, œuvres d'art et objets techniques, évoquant aussi bien le Futurisme de Léger et Marinetti que certaines œuvres de Le Corbusier, mais aussi la Tour Eiffel, la Joconde, des moteurs comme celui de la Matra, un cycliste qui utilise des clés pour réparer son vélo), ou encore la pratique du sport, pour expliquer en quoi consiste l'expérience techno-esthétique ${ }^{17}$ :

[...] la techno-esthétique n'a pas pour catégorie principale la contemplation. C'est dans l'usage, dans l'action, qu'elle devient en quelque sorte orgasmique, moyen tactile et moteur de stimulation. Quand un écrou bloqué se débloque, on éprouve un plaisir moteur, une certaine joie instrumentalisée, une communication, médiatisée par l'outil, avec la chose sur laquelle il opère. C'est un type d'intuition perceptivomotrice et sensorielle. Le corps de l'opérateur donne et reçoit ${ }^{18}$.

Ce que Simondon et Dewey thématisent, ce n'est pas seulement le prolongement de la vie dans des artefacts techniques, c'est aussi la "jouissance », l'engagement d'un corps entièrement ouvert et sensible qui, dans ce prolongement, peut se manifester en faisant 
ainsi émerger, de manière plus intense un "sens de la vie immédiate ». L'esthétique à l'état brut de Dewey, tout comme la techno-esthétique de Simondon, est donc une réflexion qui considère l'opérer technique de l'homme en tant que capacité à faire sentir l'expansion de la vie, de la sensibilité, de l'aesthesis, à travers des individuations de plus en plus organisées et complexes.

23 Ni l'esthétique de Simondon ni celle de Dewey ne sont, par conséquent, proches d'une forme d'esthétique substantialiste : elles peuvent être considérées au contraire, et selon le mot de Gilbert Hottois, comme des formes de « sensibilité à ce qui est produit ${ }^{19}$ ». Les réflexions de Simondon et de Dewey font de la relation techno-esthétique de l'homme à ses artefacts, où est en jeu la réorganisation du milieu humain et donc de l'aesthesis humaine, un rapport absolument non contemplatif, mais toujours intrinsèquement trans-actif. Il apparaîtra certes que le parallèle entre la techno-esthétique de Simondon et l'esthétique de Dewey a des limites. Dewey considère pour sa part le caractère intensifié de l'expérience vitale comme étant éminemment actif dans les œuvres d'art : c'est précisément la thèse qui caractérise un livre comme L'art comme expérience. Selon lui, l'art est par excellence la modalité d'une expérience bien formée. Dewey, tout au contraire de Simondon, a parfois tendance à oublier que la bonne expérience, le fait de sentir, donc, cette intensification de la vie, est tout d'abord une manière de vérifier que l'expansion de la vie humaine se sert d'artefacts techniques. Ainsi l'ambiguïté de son attitude à l'égard de la technique devra-t-elle être signalée, même si sa convergence plus large avec la techno-esthétique de Simondon reste bien l'essentiel.

Dewey pense la technique comme un élément constitutif de la vie humaine, dans la mesure où l'interaction de l'organisme humain avec le milieu lui semble indissociable des médiations à caractère technique. En témoignent les propos d'Expérience et nature sur l'instrument technique. Ici, Simondon et Dewey s'opposent conjointement à tout type de formulation dualiste et métaphysique - ce qui vaut déjà dans le rapport de l'individu au milieu. Il n'y a jamais une réelle opposition entre sujet et objet. Tout comme dans Du mode d'existence des objets techniques, où Simondon a "l'intention de susciter une prise de conscience du sens des objets techniques » pour montrer que "l'opposition dressée entre la culture et la technique, entre l'homme et la machine, est fausse et sans fondement ${ }^{20}$ ", Dewey apparaît animé par l'exigence de se débarrasser d'une barrière métaphysique, imposée par une certaine tradition de pensée, entre homme et objets techniques. La technicité de Du mode d'existence des objets techniques est l'objet d'une enquête en tant que forme transactive et « réalité riche en efforts humains et en forces naturelles, qui constitue le monde des objets techniques, médiateurs entre la nature et l'homme ${ }^{21}$ ». De la même manière, Dewey conçoit le faire technique autrement qu'un pur processus d'application, puisqu'il est un moyen de résolution mais aussi de transformation du rapport entre l'organisme et le milieu :

[...] un outil est une chose particulière, mais en même temps beaucoup plus de cela, puisqu'il renferme une connexion, une liaison séquentielle naturelle. [...] Les préjugés que l'homme nourrit envers lui-même le conduisent aisément à ne considérer un outil que par rapport à lui, à sa main et ses yeux, mais la relation primitive est celle qui l'unit à d'autres choses extérieures, comme le marteau au clou et la charrue à la terre. Seul ce lien objectif le place en relation avec l'homme lui-même et ses activités. Tout outil renvoie à la perception et à la reconnaissance de liens séquentiels dans la nature ${ }^{22}$. 
[...] qui est une condition de possibilité du fonctionnement de l'objet technique. L'objet technique est donc la condition de lui-même comme condition d'existence de ce milieu mixte, technique et géographique à la fois ${ }^{23} »$. Le type de lien fortement processuel et opérationnel que Simondon reconnaît est tel grâce au mode particulier d'existence des objets techniques, qui devrait avoir en soi une certaine indétermination, en pouvant donc conserver une forte marge d'indétermination, et en restant ainsi ouvert à la contingence: "le véritable perfectionnement des machines, celui dont on peut dire qu'il élève le degré de technicité, correspond non pas à un accroissement de l'automatisme, mais au contraire au fait que le fonctionnement d'une machine recèle une certaine marge d'indétermination. C'est cette marge qui permet à la machine d'être sensible à une information extérieure ${ }^{24} »$. Dewey, à sa façon, reconnaît cette ouverture de l'instrument comme devenir :

[...] les choses détiennent des potentialités ou sont des instruments, non parce qu'elles Sont, mais plutôt parce qu'elles Sont en voie d'être. Elles conduisent ellesmêmes à des connexions opératoires par lesquelles elles s'accomplissent, du fait qu'elles ne sont pas elles-mêmes réelles au sens propre du terme. Ce point de vue a permis à la pensée grecque d'éviter l'unilatéralité moderne qui conçoit les outils comme de pures commodités subjectives ${ }^{25}$.

On peut bien reconnaitre dans ces mots de Dewey l'un des points les plus significatifs de l'élaboration simondonienne d'une pensée de la technique : celui selon lequel l'objet individuel n'est pas telle ou telle chose donnée hic et nunc, mais ce dont il y a genèse ${ }^{26}$.

Lorsque le discours de Dewey passe de l'analyse de l'esthétique à l'état brut à celle de l'esthétique raffinée par l'art, sa conception de la technique semble plus conventionnelle. Il semble presque que, dans L'art comme expérience, l'esthétique tende à écarter le domaine de l'opération technique pour se concentrer exclusivement sur le domaine artistique. Ce possible glissement interprétatif me semble lié au fait que Dewey enracine à nouveau l'art dans la nature. Son intention est en effet de démontrer l'enracinement naturel des arts et de l'expérience esthétique: "on identifie généralement l'œuvre d'art à l'édifice, au livre, au tableau ou à la statue dont l'existence se situe en marge de l'expérience humaine. Puisque la véritable œuvre d'art se compose en fait des actions et des effets de ce produit sur l'expérience, cette identification ne favorise pas la compréhension ${ }^{27}$ ». Dewey soutient ici que si l'on veut comprendre l'art, on ne doit pas considérer exclusivement les produits liés à l'art conventionnel, ceux du musée par exemple; on doit plutôt revenir à "la matière brute» de l'expérience, aux activités quotidiennes dans le monde, qui ne sont pas éminemment artistiques. C'est seulement de cette façon qu'on peut ressentir les arts comme une expansion et intensification de la vie, caractérisée par son enracinement dans un milieu.

Ce qui me paraît pourtant le plus significatif, ce sont les considérations déjà évoquées de Dewey sur les instruments, ces derniers étant caractérisés de telle manière qu'il est finalement possible de reconnaître que l'esthétique de Dewey reste intrinsèquement liée à la question du rapport entre vie et technique, tout comme l'est la technoesthétique simondonienne. L'expérience esthétique, telle qu'elle est formulée par Dewey, active des processus d'apprentissage technique en éduquant la sensibilité afin qu'elle expérimente des modalités innovatrices d'échange avec le monde. C'est là le sens profond de ces mots de Dewey: 
[...] « application » est un mot que beaucoup répugnent à employer. Il suppose un outil extérieur, tout prêt, complet. [...]. Une application, dans l'usage que les hommes en font, signifiant une commodité, une jouissance et une amélioration, la conception qui en fait quelque chose d'externe et d'arbitraire ne fait que refléter et renforcer les théories qui détachent l'homme de la nature ou qui, dans le langage de la philosophie, opposent le sujet et l'objet. Si toutefois nous nous affranchissons de toute préconception, [...] l'application dans quelque chose signifie une interaction [maintenant on peut dire une trans-action, une trans-duction] plus étendue des événements naturels les uns par rapport aux autres, une élimination de la distance et des obstacles, et une provision de possibilités en vue d'interactions révélant des potentialités antérieurement cachées et donnant naissance à de nouvelles histoires avec de nouveaux tenants et de nouveaux aboutissants ${ }^{28}$.

C'est précisément dans ces remarques finales que se révèlent tout le sens de la technoesthétique élaborée par Simondon ainsi que celui la réflexion esthétique de Dewey : les deux philosophes cherchent à penser le rapport de l'homme à son milieu comme ce en quoi on peut découvrir de nouvelles potentialités, de nouvelles émergences possibles du sensible; cela a lieu de manière encore plus complexe et productive grâce aux artefacts, et c'est précisément en vertu de la technique que l'adaptation spécifique de l'homme au milieu - qui n'est pas passive mais tout au contraire fortement active et transformatrice pour l'aisthesis de l'homme comme pour son milieu - acquiert sa forme caractéristique d'une expansion créatrice et inventive.

\section{BIBLIOGRAPHIE}

Barthélémy Jean-Hugues, Bontems Vincent, « Philosophie de la nature et artefact. La question du préindividuel », Appareil [en ligne], 1, 2008 : http ://appareil.revues.org/72

Barthélémy Jean-Hugues, Simondon ou l'encyclopédisme génétique, Paris, Puf, 2008

Dewey John, Knowing and the Known, in The Later Works, 1925-1953, vol. 16, 1949-1952, Southern

University Press, 1989

Dewey John, L'art comme expérience, trad. fr. J.-P. Cometti et al., Paris, Folio, 2010

Dewey John, Expérience et nature, trad. fr. J. Zask, Paris, Gallimard, 2012

Hottois Gilbert, Technoscience et technoesthétique chez Gilbert Simondon, in P. Chabot, Simondon, Paris, Vrin, 2002

Montani Pietro, Esperienza estetica e anestesie dell'esperienza, in Esperienza estetica a partire da John Dewey, Æsthetica prepint, Palermo, Centro Internazionale Studi di Estetica, 2007

Montani Pietro, Tecnologie della sensibilità. Estetica e immaginazione interattiva, Raffaello Cortina, Milano, 2014

Montani Pietro, Bioesthétique. Sens commun, technique et art à l'âge de la globalisation, Paris, Vrin, 2014

Simondon Gilbert, Du mode d'existence des objets techniques, Paris, Aubier, 1958. 
Simondon Gilbert, L'individuation à la lumière des notions de forme et d'information, Grenoble, Éditions Jérôme Millon, 2005.

Simondon Gilbert, Sur la techno-esthétique, in Sur la technique (1953-1983), Paris, Puf, 2014.

\section{NOTES}

1. Tandis qu'ici on ne considère pas la réflexion esthétique seulement du point de vue de l'enquête sur l'art. Que la relation existant entre l'art et la technique présente, en elle-même, un caractère constitutif paraît un point significatif. Dans la Grèce antique, le mot téchnè signifiait art et technique; c'est seulement à l'époque moderne, avec la naissance de l'esthétique dans l'ouvrage de A. G. Baumgarten (Aesthetica, 1750), que la possibilité de leur dissociation, menant à des formes de différends comme d'alliances entre art et technique, a été esquissée. Ce sont ces différends et ces alliances qui ont caractérisé la réflexion sur l'esthétique jusqu'à nos jours.

2. Nous faisons ici référence aux réflexions sur l'esthétique élaborées, en Italie, par les philosophes Emilio Garroni et Pietro Montani. Le présent article doit beaucoup aux ouvrages de Pietro Montani, : Bioestetica. Senso comune, tecnica e arte nell'età della glocalizzazione (Roma, Carocci, 2007) - dont la traduction française Bioesthétique. Sens commun, technique et art à l'âge de la globalisation a été publiée chez Vrin en 2014 -, et Tecnologie della sensibilità. Estetica e immaginazione interattiva (Milano, Raffaello Cortina, 2014).

3. Gilbert Simondon, Sur la techno-esthétique, in Sur la technique (1953-1983), Paris, Puf, 2014, p. 391-392.

4. John Dewey (Burlington 1859-New York 1952) est considéré comme l'un des plus importants membres de l'école pragmatiste américaine. Parmi ses références, il faut mentionner W. James et C. S. Peirce ; Dewey était un philosophe animé par des intérêts très divers, de l'épistémologie à la pédagogie, en passant par la théorie politique et l'esthétique : sa riche production théorique en témoigne.

5. G. Simondon, L'individuation à la lumière des notions de forme et d'information, Grenoble, Editions Jérôme Millon, 2005, p. 25.

6. Jean-Hugues Barthélémy \& Vincent Bontems, «Philosophie de la nature et artefact. La question du préindividuel », Appareil [en ligne], 1, $2008:$ http ://appareil.revues.org/72

7. G. Simondon, L'individuation à la lumière des notions de forme et d'information, op. cit., p. 33.

8. Ibid., p. 9.

9. Ibid., p. 538. Sur le caractère einsteinien du schème physique de champ chez Simondon, voir Jean-Hugues Barthélémy, Simondon ou l'encyclopédisme génétique, Paris, Puf, 2008. Einstein avait lui-même écrit que le concept de champ était le concept le plus important de la physique depuis Newton.

10. J. Dewey, Knowing and the Known, in The Later Works, 1925-1953, vol.16, 1949-1952, Southern Illinois University Press, 1989, p. 96-97.

11. Ibid., p. 101-102.

12. Ibid., p. 114.

13. «[...] l'esthétique de Dewey est d'abord une esthétique physiologique, qui place au centre de sa réflexion le rapport déterminant entre organisme et milieu ", P. Montani, Esperienza estetica e anestesie dell'esperienza, dans Esperienza estetica a partire da John Dewey, Palermo, Æsthetica prepint, Centro Internazionale Studi di Estetica, 2007. (nus traduisons)

14. Ibid., p. 243.

15. J. Dewey, L'Art comme expérience, trad. fr. J.-P. Cometti et al., Paris, Folio, 2010, p. 31-32.

16. Ibid., p. 32 . 
17. Rappelons que Simondon, au début sa la Lettre, écrit que «[...] s'il s'agit de régénérer la philosophie contemporaine, il faut songer de manière privilégiée aux interfaces, [...] il faut faire aussi appel à la pensée et aux réalisations, pensées réflexivement ou non, de l'esthétique. Pourquoi ne pas penser à la fondation et peut-être à une axiomatisation provisoire d'une esthétotechnique ou techno-esthétique? ", Sur la techno-esthétique, op. cit., p. 380.

18. Ibid., p. 383.

19. G. Hottois, "Technoscience et technoesthétique chez Gilbert Simondon », in P. Chabot, Simondon, Paris, Vrin, 2002, p. 104.

20. G. Simondon, Du mode d'existence des objets techniques, Paris, Aubier, 1958, p. 9.

21. Ibid., p. 9.

22. J. Dewey, Expérience et nature, trad. fr. J. Zask, Paris, Gallimard, 2012, p. 122.

23. G. Simondon, Du mode d'existence des objets techniques, op. cit., p. 55.

24. Ibid., p. 11.

25. J. Dewey, Expérience et nature, op. cit., p. 122.

26. G. Simondon, Du mode d'existence des objets techniques, op. cit., p. 20.

27. J. Dewey, L'art comme expérience, op. cit., p. 29.

28. J. Dewey, Expérience et nature, op. cit., p. 156.

\section{RÉSUMÉS}

En 1982, Gilbert Simondon écrit une lettre, jamais envoyée, à Jacques Derrida. Dans ces pages émerge le concept fondamental de techno-esthétique. La réflexion esthétique que Simondon y esquisse consiste en une reprise et une radicalisation de ce qu'il avait déjà théorisé dans Du mode d'existence des objets techniques, à propos du rapport très strict que l'on peut envisager entre technique et esthétique : la sensibilité humaine a toujours été connectée avec des prolongements techniques par lesquels cette sensibilité de l'individu se réorganise, tout en réorganisant ce qui l'entoure. Cet article vise à montrer que ce type de conception techno-esthétique est également présent dans certains textes de John Dewey. La convergence entre les deux auteurs s'exprime également dans leur façon de considérer le rapport, que l'on peut qualifier de co-constitution, entre l'organisme et son milieu ; tout comme pour Simondon, qui développe la notion de « milieu associé », il n'est possible, pour Dewey, de concevoir un individu qu'à partir de sa relation à un milieu. Par là, c'est le parallélisme entre les notions de «transduction » et de «transaction » qui sera à explorer.

\section{INDEX}

Mots-clés : Dewey, esthétique, Simondon, technique, techno-esthétique, transaction, transduction 


\section{AUTEUR}

\section{ELISA BINDA}

Doctorante en philosophie à l'université La Sapienza de Rome. Elle s'intéresse surtout aux aspects esthétiques de la philosophie de Gilbert Simondon. elisa.binda@uniroma1.it 